Cahiers de géographie du Québec

\title{
La territorialisation à l'épreuve des temps
}

Mobilisation du temps et des temporalités dans la

territorialisation des politiques environnementales

Territorialisation: Standing the Test of Time

\section{The Use of Time and Temporality in the Territorialisation of} Environmental Policies

\section{Olivier Alexandre et Coralie Mounet}

Volume 52, numéro 145, avril 2008

URI : https://id.erudit.org/iderudit/018425ar

DOI : https://doi.org/10.7202/018425ar

Aller au sommaire du numéro

Éditeur(s)

Département de géographie de l'Université Laval

ISSN

0007-9766 (imprimé)

1708-8968 (numérique)

Découvrir la revue

Citer cet article

Alexandre, O. \& Mounet, C. (2008). La territorialisation à l'épreuve des temps : mobilisation du temps et des temporalités dans la territorialisation des politiques environnementales. Cahiers de géographie du Québec, 52(145), 9-26. https://doi.org/10.7202/018425ar

\section{Résumé de l'article}

Explorant le lien indéfectible qui existe entre temps et espace, entre histoire et géographie, cet article s'intéresse aux manières dont le temps physique et le temps représenté - les temporalités - peuvent effectivement constituer des éléments-clés de la construction des territoires et devenir des outils utilisés par les acteurs. Deux types de crise environnementale servent de point de départ à cette réflexion : le retour du loup dans les espaces ruraux français et la sécheresse généralisée au Maroc. À partir de ces deux contextes radicalement différents, la construction d'une grille méthodologique permet de montrer les fondements épistémologiques des postures adoptées, au sein des conflits sur le sens et la fonction d'un territoire. Les divergences entre des conceptions du territoire dans le temps y sont centrales. Et pour y remédier, l'action publique se saisit plus ou moins implicitement de l'outil temporel. 


\section{La territorialisation à l'épreuve des temps}

\section{Mobilisation du temps et des temporalités dans la territorialisation des politiques environnementales 1}

\author{
Territorialisation: Standing the Test of Time \\ The Use of Time and Temporality in the \\ Territorialisation of Environmental Policies
}

Olivier ALEXANDRE et Coralie MOUNET

Université Joseph Fourier

Alexandreolivier@yahoo.fr

Coraliemounet@hotmail.com

\section{Résumé}

Explorant le lien indéfectible qui existe entre temps et espace, entre histoire et géographie, cet article s'intéresse aux manières dont le temps physique et le temps représenté - les temporalités - peuvent effectivement constituer des éléments-clés de la construction des territoires et devenir des outils utilisés par les acteurs. Deux types de crise environnementale servent de point de départ à cette réflexion: le retour du loup dans les espaces ruraux français et la sécheresse généralisée au Maroc. À partir de ces deux contextes radicalement différents, la construction d'une grille méthodologique permet de montrer les fondements épistémologiques des postures adoptées, au sein des conflits sur le sens et la fonction d'un territoire. Les divergences entre des conceptions du territoire dans le temps y sont centrales. Et pour y remédier, l'action publique se saisit plus ou moins implicitement de l'outil temporel.

\section{Mots-clés}

Temps, temporalités, territoire, action publique, politique environnementale, gestion de la faune sauvage, gestion de l'eau

\section{Abstract}

This paper examines links between time and space and between history and geography by observing the ways in which real and perceived time - which we here call temporality are central components in the construction of territories and can be used as tools by stakeholders. Two types of environmental events are taken as the starting points of our approach: the 'return' of the wolf to the French countryside and Morocco's country-wide 'drought.' Based on these dissimilar situations, a methodological grid is constructed and the epistemological bases of the approach are shown within the context of disagreement over the meaning and function of territory. Conflict between differing concepts of territory over time are at the heart of the problem. To overcome this clash, public action has more or less implicit recourse to the concept of temporality.

\section{Keywords}

Time, temporality, territory, space, public action, environmental policy, wildlife management, water management 
Aménagement ou protection du milieu naturel, la lecture de l'action sur l'environnement met traditionnellement en scène des rationalités d'acteurs diverses et, parfois, contradictoires. C'est le cas par exemple entre aménageurs et population locale ou entre protecteurs et exploitants de la nature. À partir de deux exemples, ce texte traite des modalités d'émergence et de résolution d'une crise dans les systèmes d'exploitation des ressources naturelles ${ }^{2}$ : exploitation agricole, exploitation des ressources en eau. Il s'intéresse en effet à la pénurie en eau, relative aux usages de la ressource, et aux bouleversements des pratiques d'élevage, aux conflits qu'engendre la présence du loup dans les espaces ruraux.

Pour les décrypter, la lecture adoptée ici consiste à considérer comme deux systèmes articulés les pratiques humaines et l'environnement, la crise révélant un soudain décalage entre eux. C'est la perception d'une rupture dans le système environnemental (le retour du loup, la sécheresse) qui crée cette situation et rend les pratiques plus ou moins brutalement inadaptées au contexte. Il y a alors nécessité d'actualiser à nouveau les deux systèmes et de redéfinir pour cela les modalités de régulation de leurs pratiques. Mais la crise provenant de ce décalage se complexifie et se transforme en conflit ${ }^{3}$ en raison d'une confrontation de représentations divergentes des dispositions prises pour la résoudre : deux représentations différentes du territoire dans le temps s'affrontent simultanément au sujet des conditions d'actualisation des systèmes. Les uns sont partisans de l'adaptation de l'environnement aux pratiques humaines, alors que les autres soutiennent un changement des pratiques humaines en fonction des contraintes environnementales.

Face à cette disjonction dont elle peut être le moteur, l'action publique cherche à activer de nouvelles modalités du faire-ensemble au sein du territoire. Or l'étude des cas présentés montre que, de manière parfois explicite, le temps figure comme un des éléments majeurs sur lequel elle procède à une tentative de conjonction des représentations du territoire dans le futur. À ce titre, cet article ne s'attache pas seulement à envisager la durée nécessaire à l'épaisseur temporelle, au temps cristallisé du territoire (Marié, 1982). L'originalité de la démarche tient à considérer le temps comme un instrument mobilisé par les protagonistes pour justifier leur posture au sein des conflits territoriaux. Plus que l'investissement de l'espace par les acteurs, largement étudié au détriment du temps (Bertrand et Bertrand, 2000), c'est donc leur investissement du temps qui est étudié, pour comprendre le processus de territorialisation.

A priori, le temps apparaît tout au long du processus d'émergence et de résolution du conflit : comme vecteur de conflit et comme outil pour sa résolution. Il reste à déterminer si les natures de temps mobilisées (temporalités des groupes sociaux ${ }^{4}$ ou temps physique, par exemple) jouent des rôles distincts dans ces processus. De là, quel rôle peut-on réserver au temps dans les processus de transformation du territoire?

Pour répondre à une telle question, la comparaison de ces deux cas est éclairante. Elle s'est construite sur le constat d'une similarité dans l'organisation du conflit, entre des pratiques existantes et des bonnes pratiques édictées par les pouvoirs publics. Mais c'est surtout l'identification de mécanismes analogues, dans des situations différentes (les contextes socioéconomiques et culturels, la nature des problèmes, les acteurs mobilisés), qui peut permettre de définir les éléments structurants du problème temps. 
Pour mettre en évidence cette convocation du temps, ce texte détaille tout d'abord l'articulation théorique proposée entre le territoire, sa conception et les représentations du temps. Les ancrages culturels des pratiques déterminent pour partie les types de conception du territoire et expliquent la complexité qu'introduit leur coexistence. Les mettre en évidence va permettre ensuite de montrer, dans chaque cas étudié, les divergences de temporalité créatrices de conflit entre différents groupes sociaux. C'est à ces conflits que réagit l'action publique en mobilisant un temps dont l'emploi est destiné à faire converger pratiques et représentations.

\section{Le contexte de deux problématiques spécifiques}

\section{La problématique de l'eau au Maroc}

Les sécheresses chroniques qui frappent le Maroc depuis les années 1980 y ont précipité une réforme de la politique de l'eau, conduisant l'État à ne plus dissocier les usages agricoles de la ressource, pourtant fer de lance du développement national, des autres usages, urbains ou ruraux, domestiques ou industriels. La sécheresse, devenue structurelle, nécessite en effet une remise en cause de la gestion par l'offre, à l'œuvre depuis l'époque du protectorat français ; et aujourd'hui, la perspective à 15 ans d'une pénurie généralisée apparaît comme le premier facteur de changement. Celui-ci se traduit par la volonté de coordonner les différents secteurs consommateurs d'eau au sein du réseau de grande hydraulique; il va même plus loin en affichant la volonté de contrôler l'ensemble des pratiques spatiales (notamment agricoles) pouvant interagir avec la mobilisation de l'eau. La lutte engagée contre l'envasement des barrages, par exemple, exige de réguler l'occupation des sols dans les bassins versants.

Le champ concerné par la nouvelle économie de l'eau est ainsi élargi à l'ensemble des pratiques spatiales ayant un impact sur l'hydrosphère, et articule de nouveaux acteurs, dont les représentations de la ressource peuvent diverger. Schématiquement, sept grands opérateurs se sont répartis la gestion de la ressource. À chacun correspondent une aire d'action et un mode de gestion, parfois exclusifs. Localement, l'Office national de l'eau potable (ONEP) vend l'eau aux régies municipales (ou délégataires) et la distribue aux bourgs ruraux. Les Offices régionaux de mise en valeur agricole (ORMVA), qui gèrent les grands périmètres irrigués, se partagent la ressource des grands barrages avec les grandes villes. Cette ressource est produite et vendue par les agences de bassin, mises en place progressivement depuis 2001. Celles-ci ont aussi pour mission l'adoption des bonnes pratiques de gestion, c'est-à-dire la concertation entre ces opérateurs et l'intégration des administrations ayant en charge le développement rural (Directions provinciales de l'équipement, Directions provinciales de l'agriculture) et la protection des bassins versants (Administration des eaux et forêts) ${ }^{5}$.

La gestion locale de l'eau a pour objectif de mieux articuler des espaces marqués par des formes d'usage différenciées. Ainsi, la croissance de la consommation en eau urbaine ne pose pas seulement des problèmes de concurrence à l'irrigation, mais plutôt d'extrême polarisation de la consommation. Parallèlement, la dispersion des populations rurales se heurte à l'absence de couverture du territoire par le réseau d'eau potable. À l'image d'un Maroc qui cherche à dépasser une forme de développement autour de pôles privilégiés et mis en réseaux (les grandes villes de la côte atlantique, 
les périmètres d'irrigation moderne), l'organisation de la gestion de l'eau est confrontée à la nécessité de faire émerger un nouveau territoire: un territoire où pourrait se constituer une continuité dans les infrastructures de mise en valeur de l'espace. Les stratégies de gestion deviennent ainsi centrales dans l'organisation des territoires hydrauliques. La loi sur l'eau de 1995 a été la première traduction de ce changement. Elle répond également à un processus de modernisation de l'État et de réorganisation du territoire marocain. De ce fait, elle participe, avec les nouvelles orientations des plans d'aménagement du territoire, à une actualisation idéologique correspondant aux nouveaux besoins et objectifs du développement économique et social.

\section{La problématique du loup en France}

Depuis son retour en 1992 et après une cinquantaine d'années d'absence en France, le loup montre une progression numérique et spatiale sur l'ensemble des Alpes françaises. Sa présence dans des zones rurales ou montagnardes, concernées par l'élevage, ne va pas sans dégâts aux troupeaux d'ovins, de caprins, parfois de bovins. Les pratiques d'élevage ont en effet évolué de telle sorte qu'elles ne sont plus adaptées aujourd'hui à la présence d'un prédateur. Or le contexte juridique et sociétal a également changé dans un sens beaucoup plus favorable à la protection de la nature: la gestion du dossier loup est ainsi fortement conditionnée par le cadre juridique imposant la protection stricte de cette espèce. Il s'agit par conséquent, pour les pouvoirs publics, de trouver des solutions répondant à la volonté de protéger ce prédateur, mais également à la nécessité de rendre les pratiques d'élevage plus compatibles avec sa présence. La gestion nationale du dossier et son application locale enrôle (au sens de Callon, 1992) de nombreux acteurs alimentant, par des controverses, un conflit les opposant: deux camps se sont constitués, dès les premiers temps, confrontant les détracteurs de l'animal ou lycophobes, constitués majoritairement du monde agricole et les partisans de l'animal ou lycophiles, représentés principalement par les associations de protection de la nature et les gestionnaires. Les uns, considérant la présence du prédateur comme une réelle entrave à leur profession, désirent son éradication alors que les autres, prenant l'animal comme un symbole de richesse des milieux naturels, souhaitent son maintien et son développement. Ce sont donc des enjeux contradictoires et des représentations différentes du bon rapport à la nature qui s'affrontent dans le conflit autour de la gestion du loup. Notons ici que si ce schéma d'opposition est récurrent chez la plupart des acteurs enrôlés dans ce conflit, des conditions et des opinions spécifiques à chaque territoire et plus complexes peuvent être identifiées ${ }^{6}$.

Depuis une dizaine d'années, des solutions nationales (deux programmes: L'instrument financier européen - LIFE - de 3 ans et un plan national de 4 ans) sont proposées pour aider les éleveurs et les bergers à adapter leurs pratiques à cette nouvelle contrainte environnementale: outre l'indemnisation des bêtes tuées, des mesures de protection des troupeaux existent. Pour mettre en place le gardiennage serré préconisé, des aide-bergers sont payés, des chiens de protection sont insérés dans les troupeaux et des parcs mobiles sont financés pour effectuer des regroupements nocturnes. Loin d'être anecdotiques, ces changements de pratiques mis de l'avant par l'État représentent une petite révolution pour le monde de l'élevage qui doit penser de manière différente son fonctionnement. À ces solutions, s'est ajoutée récemment 
une gestion directe du loup autour des troupeaux (prélèvement d'un certain nombre de loups chaque année et autorisation sous certaines conditions aux bergers d'effaroucher voire de tirer sur des loups à proximité des troupeaux).

L'application locale de cette gestion nationale peut être facilitée par des innovations émanant de divers acteurs. Des gestionnaires (par exemple, le Parc naturel régional et la Réserve naturelle du Vercors apportant notamment plus de proximité relationnelle et permettant une gestion proche de la concertation) ou des associations (des bénévoles auprès des bergers, par exemple) peuvent endosser le rôle de médiateur et opérer des traductions locales, aidant à un meilleur vivre-ensemble.

\section{Territoire apodictique, territoire assertorique: deux conceptions à l'origine d'une crise territoriale}

Les deux cas choisis comme exemple se caractérisent par la volonté d'adapter les pratiques sociales à un contexte naturel caractérisé par un changement récent ${ }^{7}$. Dans un cas, la présence du loup met en scène trois grands groupes d'acteurs aux positions distinctes. Les associations de protection de la nature, partisanes d'un changement des pratiques agricoles qui permette de pérenniser le retour du loup, se confrontent au monde agricole pour qui les attaques de loup sur les brebis représentent une contrainte inacceptable, dont la société française s'est difficilement affranchie. L'action publique joue face à cela un rôle de régulateur et cherche la formulation d'un compromis entre les deux parties.

Dans le second cas, la sécheresse structurelle connue par le Maroc sert d'alibi à la réorganisation du secteur de l'eau et au repositionnement de l'État vis-à-vis des opérateurs publics; ceux-ci ont en effet favorisé le fractionnement de la ressource par secteurs d'usage concurrents, aggravant l'impact de sa rareté. Entre l'action publique des opérateurs locaux et les politiques sectorielles des ministères de tutelle, s'immiscent peu à peu les agences de bassin hydraulique, acteur régional porteur d'une pratique intégrée de la gestion.

Ces oppositions, à propos des pratiques sur le territoire, traduisent un processus qui dépasse le simple conflit entre des rationalités diverses. La crise du système des pratiques met en scène deux conceptions de sa réactualisation.

La première s'appuie sur une transformation du territoire existant: d'une part, la mise en place de nouvelles pratiques agricoles sur les zones de présence du loup (orientation favorisée par les pouvoirs publics et relayée par les lycophiles); d'autre part, la définition de territoires hydrauliques à travers l'émergence du bassin versant comme unité principale de gestion de l'eau et de ses usages (vision portée par la nouvelle politique de l'eau et relayée par les agences de bassin).

La seconde conception revendique au contraire la prégnance des caractéristiques du territoire existant dans la formulation de la régulation (vision partagée, dans le cas de l'eau, par l'ensemble des opérateurs locaux qui revendiquent une légitimité territoriale spécifique et, dans le cas du loup, par les lycophobes arguant notamment de leur ancrage territorial). 


\section{Quelle conception légitime du territoire pour agir?}

C'est bien un décalage de la conception du territoire dans le temps que traduit cette opposition. Pour l'analyser, posons l'hypothèse que ces deux conceptions s'appuient sur deux manières de penser le territoire, l'un prescriptif et l'autre hérité. Dans le premier cas, le (nouveau) territoire est l'expression de l'actualisation des pratiques par rapport au milieu et représente l'expression d'un espace d'intervention censé assurer l'appropriation par les citoyens/usagers des pratiques et du territoire résultant. Le territoire est consécutif de la production d'un savoir normatif fondant une diachronie entre un agencement spatial passé et l'organisation des pratiques spatiales dans le présent de l'action. Il correspond à une dimension projective du territoire, c'est-à-dire une dimension qui s'inscrit dans un temps futur et qui tente de plier la réalité à un modèle de développement. Il tend à la coexistence, d'une part de l'avant et de l'après, et d'autre part, d'un environnement réel et d'un environnement idéalisé.

Dans le second cas, la conception du territoire hérité repose moins sur la seule dimension politique que sur le rapport du collectif à l'espace. Dans les discours, elle renvoie aussi à l'inscription spatiale d'un processus structurant et devenu endogène, collectivement reconnu comme tel par certains groupes d'acteurs et effectif de manière continue sur un espace donné.

Ces deux conceptions schématiques transparaissent effectivement dans la disposition des conflits étudiés.

Dans la construction de la gestion de l'eau au Maroc, les tentatives successives de transfert des compétences aux différents acteurs renvoient d'abord à la distribution territoriale de la responsabilité de la gestion: de l'État aux associations d'usagers, en passant par les opérateurs nationaux, les agences de bassins et les opérateurs locaux, l'énonciation des bonnes pratiques dans le territoire se justifie à plusieurs échelles. Mais à cette recherche de la bonne échelle du territoire de gestion correspond ainsi une hésitation entre les deux approches. Du côté de l'État, la prise en compte du local se fait de manière normative et prescriptive, par l'incitation des usagers ruraux et agricoles à se constituer en associations, par exemple. Par ailleurs, les résistances des opérateurs locaux renvoient à une conception empirique du territoire, comme héritage. Aussi, après que le monde rural se soit opposé aux pratiques d’ingénierie portées par les administrations d'État (génie forestier, agricole ou hydraulicien), la délégation aux associations d'usagers de l'initiative de certains programmes d'aménagement et de la gestion de certains réseaux pose de nouveaux problèmes: la prise en compte des pratiques locales, nouveau paradigme de l'action publique, constitue un défi nécessitant une redéfinition de l'action de l'administration. L'État tente, dans ce contexte, d'imposer une nouvelle conception du territoire qui ne correspond pas à la forte inscription territoriale de l'action des opérateurs locaux, notamment dans les périmètres d'irrigation moderne. En outre, cette conception remet en cause, à l'avenir, la légitimité de leur action. Si les spécificités locales sont ainsi mises en avant, elles se heurtent à l'héritage de pratiques étatiques anciennes et incarnées par les opérateurs. Enfin, la dimension générique de la prise en compte du niveau local ne correspond pas forcément aux attentes endogènes exprimées par les habitants.

Concernant la gestion du loup, l'analyse de la perception qu'en ont les lycophobes directement concernés, dans les premières années du conflit, montre une opposition similaire. Le monde agricole a effectivement développé un argumentaire de 
l'appartenance à un territoire local pour légitimer sa position: ses pratiques sont bonnes car spécifiques à son territoire, leur légitimité vient de leur caractère endogène et hérité. Face à cela, le monde des protecteurs de la nature régulièrement associé à l'action publique (Lascoumes, 1994) est considéré comme extérieur au territoire. Le changement des pratiques que ceux-ci préconisent et imposent comme solution (en ce qui concerne l'action publique) est perçu comme exogène et générique.

\section{Les rapports du territoire aux temps: une grille de lecture de la complexité}

La coexistence de ces deux conceptions peut être interprétée comme l'expression d'une dualité épistémologique entre un savoir rationaliste, général, et un savoir reposant sur la description du particulier. En effet, le savoir au fondement de la territorialisation se construit sur la valorisation de caractéristiques essentielles car pérennes, parallèlement à l'émergence chronique du nouveau (Da Costa Gomès, 1997). Le territoire se retrouve ainsi dans l'articulation et la territorialisation d'un savoir à forte fonction prescriptive, censé expliquer le monde tel qu'il devrait être, et d'un savoir positif à faible fonction prescriptive, tentant d'expliquer le monde tel qu'il est. On retrouve cette dualité, formulée en d'autres termes, dans l'opposition des savoirs experts et profanes telle que la décrivent Callon, Lascoumes et Barthe (2001).

Cette articulation de territoires conçus dans différents rapports au temps compose ainsi une trame systémique dans laquelle on identifie des catégories de perception et de conception du monde. Elle va nous servir de grille de lecture pour permettre l'analyse de la crise au sein des territoires (tableau 1). Telle que nous la concevons, cette grille distingue les différents éléments constitutifs des deux conceptions divergentes; l'opposition de ces éléments crée ce que l'on peut appeler des ordres de complexité, c'est-à-dire des champs problématiques dans lesquels est susceptible de se développer la crise. Ils sont les révélateurs de la coexistence d'un référentiel apodictique (le territoire normatif) et d'un référentiel assertorique (le territoire empirique) dans les logiques d'établissement de l'action territorialisée ${ }^{8}$.

\section{Tableau 1 Deux conceptions du territoire dans le temps : diverses formes de complexité à l'origine de la crise}

\begin{tabular}{l|c|c}
\hline \multirow{2}{*}{ Territoire apodictique } & \multicolumn{1}{c}{ Territoire assertorique } \\
\cline { 2 - 3 } & Ordre de complexité & \\
\hline Projectif & Temporel & Hérité \\
Exogène & Spatial & Endogène \\
Générique & Social & Spécifique \\
Normatif & Politique & Empirique \\
\hline
\end{tabular}

La forme dialectique de cette formalisation ne doit pas faire oublier son utilisation systémique pour la prise en compte de la complexité caractérisant le système des pratiques. À l'articulation apodictique-assertorique correspondent donc des complexités distinctes. La plus évidente, d'ordre temporel, articule le maintien de l'existant (l'hérité) et la volonté de transformer l'existant par rapport à un modèle que l'on s'est 
donné (projectif). On peut ensuite distinguer une complexité d'ordre spatial, entre ce qui est généré au sein du territoire (endogène) et ce qui provient de l'extérieur (exogène), une complexité d'ordre social, entre des modalités d'action spécifiques au territoire et des modalités génériques dont la justification réside justement dans cette distance vis-à-vis des spécificités du territoire. Enfin, une complexité d'ordre politique correspond à la prise en compte du territoire par l'action publique, suivant une approche soit normative, c'est-à-dire, ici, dans une conception globalisante, soit empirique, c'est-à-dire conçue à partir de la connaissance des spécificités locales.

Cette grille d'analyse propose une distinction qui n'apparaît, bien sûr, pas aussi clairement dans la réalité du territoire; elle a avant tout une fonction heuristique. Elle sert à mettre en évidence les fondements de l'action dans le territoire pour mieux en comprendre l'implication dans l'émergence de la crise et son utilisation sur les divergences des représentations du territoire, à l'origine du conflit.

\section{Quand le temps est vecteur d'un désaccord sur le territoire}

Dans les deux cas étudiés, les divergences sont consécutives à un processus de justification de l'action territorialisée mettant en scène un territoire prescriptif et un territoire hérité. En ce qui concerne l'eau, c'est la coexistence de deux hydrauliques particulièrement structurantes qui traduisent ces représentations distinctes du territoire. Dans le cas du loup, la présence de l'animal fait s'affronter deux groupes sociaux qui conçoivent un devenir du territoire de manière radicalement opposée, malgré l'usage d'un même ordre de justification des pratiques.

\section{Le temps des territoires hydrauliques}

La problématique de l'hydraulique marocaine articule deux types de réseaux, porteurs des deux conceptions du territoire dans le temps:

- celui de la grande hydraulique, structure majeure d'initiative étatique, qui permet d'investir le marché de l'exportation par la création de grands périmètres irrigués depuis les barrages installés dans les vallées de montagne (Rif, Moyen-Atlas et Haut-Atlas) ; depuis le Protectorat, ce réseau est un des principaux outils normatifs de transformation et de développement du territoire mis en place par l'État;

- celui de la petite et moyenne hydraulique, marqué par la multitude des unités de desserte dont la variation des formes est avant tout le résultat d'une croissance locale de la demande - ou d'une baisse locale de l'offre, en période de sécheresse; ce réseau, parfois multicentenaire, répond à une logique d'autoconsommation et caractérise les territoires ruraux.

Du point de vue technique, ces deux formes de réseau n'ont pas la même implication territoriale, la première faisant émerger une entité autour du partage planifié de la ressource, tandis que l'autre résulte des évolutions spontanées du territoire. Elles ne répondent pas non plus aux mêmes phases d'évolution. En effet, le réseau de grande hydraulique s'est transformé de manière interne, consécutivement aux évolutions des modalités de production agricole (faisant intervenir des échelles propres aux réseaux de distribution des biens produits) et aux procédures d'adéquation entre les secteurs 
d'usage (l'alimentation en eau potable et l'irrigation). Cette transformation revêt par conséquent un aspect structurel qui explique que la politique de gestion de l'eau se soit d'abord cantonnée à cette infrastructure par: le contrôle des quantités d'eau distribuées, le calcul des arrosages en fonction des types de production, l'édification du réseau de distribution, la réponse à la demande.

Les réseaux locaux résultent quant à eux d'un processus basé sur une conjoncture juxtaposant la logique des besoins locaux et celle des politiques publiques dans le domaine du développement rural (généralisation progressive de l'accès à l'eau, modernisation des réseaux). Ils ont joué un grand rôle dans l'organisation sociale et spatiale des sociétés de montagne et restent un élément clé du maintien des populations dans les vallées.

Or dans le contexte de pénurie annoncée par les plus hautes autorités, le poids des espaces de grande hydraulique dans l'économie du pays conduit à reconsidérer la nature de cette distinction dans la réglementation. En effet, le contexte naturel ne rendant plus extensible la quantité d'eau mobilisable, la ressource se trouve désormais dans l'ensemble des réseaux et conduit à envisager les pratiques, dites traditionnelles, du réseau de petite hydraulique comme un aléa social menaçant la qualité et la quantité d'eau utile aux grands pôles de consommation. En reconsidérant la place de la petite et moyenne hydraulique, la politique de l'eau convoque dans son champ d'application une représentation du territoire contradictoire avec la vision très normative imposée par une action hydraulique qui est toujours restée très centralisée. Le fonctionnement de la petite et moyenne hydraulique traditionnelle apparaît dès lors comme une résistance à l'organisation territoriale d'une nouvelle économie de l'eau. De deux hydrauliques distinctes (mais qui ont toujours été complémentaires), on passe ainsi à la nécessité de gérer deux territoires dont l'un est normé alors que l'autre apparaît désormais comme à normaliser. Or cette situation est d'autant plus problématique que ces deux types de territoire incarnent deux concurrents en matière de consommation d'eau: dans la région de Marrakech par exemple, la survie de la grande hydraulique dépend de la réduction, à hauteur de $20 \%$ en 20 ans, de la consommation d'eau de la petite hydraulique (Ministère de l'équipement, 1992). En d'autres termes, la prise en compte de plus en plus forte de l'hydraulique rurale traduit un glissement des conflits noués autour du partage de la ressource: d'une dialectique rurale par opposition à urbaine à une dialectique agricole-urbaine par opposition à rurale. Et pour aborder ce conflit, la stratégie à adopter doit consister au dépassement des spécificités et logiques héritées des pratiques passées.

\section{La conception du territoire à l'origine de divergences sur la biodiversité}

La présence du loup oppose le camp des lycophiles à celui des lycophobes. Chacun de ces camps, pour justifier son positionnement, considère ses pratiques ou les pratiques qu'il préconise, comme s'insérant dans un temps long.

Les acteurs composant le camp des lycophiles (gestionnaires, protecteurs de la nature, etc.) considèrent en effet la progression du milieu naturel, dont le retour du loup fait partie, comme le résultat d'une politique de protection de l'environnement des dernières décennies. En outre, la présence de ce qu'ils nomment un superprédateur est là pour 
rendre visible le bon état de santé des milieux naturels. Le camp des lycophobes, de même, perçoit les pratiques agricoles comme ancrées fortement dans le territoire, car existantes et légitimées depuis très longtemps. L'affranchissement des contraintes environnementales (dont la menace du loup) tout comme le maintien de l'ouverture des paysages favorisé par les politiques agricoles, est en effet le résultat d'un travail humain important, perpétué sur plusieurs générations. Enfin, chacune des parties estime détenir la vérité en matière de bonnes pratiques à savoir, respectueuses de l'environnement et favorisant la biodiversité (Micoud, 1993). Ces différents camps, si l'on en croit leurs propos, ne montrent donc pas de divergence dans leurs temporalités, puisqu'ils s'insèrent tous les deux dans un temps long.

Pourtant, le retour du loup est perçu par les uns comme une continuité des processus actuels et par les autres comme une rupture brutale avec la situation antérieure. Le conflit est là, mettant bien en évidence la divergence qui existe, malgré les discours, entre les temporalités. Cette divergence est liée à des justifications de différents ordres, sous-tendues par des cultures différentes du rapport à la nature. Les lycophobes ont généralement pour référence une logique productive et des éthiques anthropocentrées (Larrère, 1997), alors que les lycophiles font plutôt appel à des logiques d'ordre récréatif ou gestionnaire et des éthiques non anthropocentrées. Or ces justifications engendrent une forte divergence des représentations de territoires dans le temps. Le monde agricole n'envisage aucun changement dans ses territorialités actuelles et passées, alors que le monde des protecteurs de la nature et des gestionnaires préconise un changement et une adaptation du système humain à la nouvelle contrainte du loup. Selon le monde agricole, la présence de ce prédateur, autrefois considéré comme un nuisible, mettrait non seulement en péril son avenir proche, mais aurait aussi pour conséquence une perte de la biodiversité produite grâce à l'élevage. À l'inverse, pour les associations de protection de la nature, la présence de cette espèce serait non seulement l'indicateur du bon état de santé des espaces naturels, synonyme de pratiques favorisant un retour de biodiversité, mais pourrait être également à l'origine de retombées économiques en matière de tourisme.

En somme, le même discours de préservation de biodiversité justifie, d'une part la défense d'un patrimoine culturel et, d'autre part, celle d'un patrimoine naturel. En ce sens, la vision du territoire par le monde des protecteurs de la nature semble être prédictive. Ces derniers préconisent un changement des pratiques du système humain, argumentant par la nécessité de protéger le patrimoine naturel. À l'inverse, le monde agricole se base sur un territoire hérité qui fait patrimoine pour envisager son histoire future.

Face aux mesures de protection proposées par l'action publique, et considérées comme la solution par les protecteurs de la nature, le monde agricole argue de la spécificité de son territoire et de l'incompatibilité des mesures de protection avec ses pratiques. Le choix du gouvernement (qui conforte en partie la position des protecteurs de la nature) de maintenir la protection du loup et de proposer des solutions pour une cohabitation est perçu par les éleveurs comme une imposition traduisant une logique d'action exogène.

Toutefois, si les premières confrontations des lycophiles et des lycophobes ont bien été celles de deux types de conception du territoire, cette situation a évolué et les catégories déterminées ici ont rapidement changé vers des positions beaucoup moins tranchées. 
Et c'est précisément dans la recherche de nouvelles convergences que le temps fait l'objet d'une instrumentalisation explicite par l'action publique. Néamoins, un temps dont la nature est autre que celle à l'origine de la crise.

\section{La mobilisation du temps par l'action publique: l'émergence de nouvelles convergences}

Face à ces divergences, l'action publique mobilise plus ou moins directement le temps pour tenter de créer les conditions d'un faire-ensemble. Pour cela, elle procède de manière radicalement opposée au Maroc et en France: dans un cas, elle énonce un nouveau référentiel temporel censé devenir commun alors que, dans l'autre cas, elle fournit véritablement du temps pour la mise en place de nouvelles pratiques.

\section{Mobiliser l'avenir pour dépasser les territoires hydrauliques}

La position tenue par la nouvelle politique de l'eau marocaine consiste à formuler un nouveau référentiel temporel, commun aux deux types d'hydraulique et de territoire. Elle s'appuie en cela sur une évolution générale de la problématique de l'eau à l'échelle internationale. Depuis les années 1980, celle-ci insiste de plus en plus sur la nécessité non seulement de répondre à la demande en eau mais de la remodeler. Parallèlement, la production d'un nouveau sens (le bien commun ou l'intérêt général) permet de redéfinir une stratégie commune aux acteurs, cadrée par une définition globalisante de la ressource. C'est sans doute rappeler une évidence que de dire que l'eau est un facteur historique de l'organisation de l'espace. Mais le développement des techniques d'alimentation, la multiplication des usages et des acteurs, l'émergence de nouvelles formes de mise en valeur, la polarisation urbaine de la consommation ont changé la nature du rapport du territoire à l'eau. Ce rapport, de plus en plus globalisant, s'inscrit dans les représentations futures du territoire.

Avec l'application progressive de la loi 10-95 sur l'eau, le Maroc investit l'exploitation de l'eau d'une forte dimension territoriale. En ce sens, elle suit un mouvement général d'élargissement sémantique de ce qui fait l'objet d'une gestion: de la ressource à l'hydrosystème d'une part, des réseaux techniques aux réseaux d'action développés pour organiser les usages d'autre part. L'enjeu de la gestion est donc de faire passer l'ensemble des acteurs locaux de l'eau du statut de communautés (vision moderne, locale, sectorielle) à celle de société (vision postmoderne, solidaire, intégrée) (Dollfus et al., 1999).

Le recours par l'État à la notion de solidarité hydraulique, à la fois au sein des bassins hydrographiques et entre ces bassins, apparaît comme le levier principal de ce passage. Elle permet de généraliser une pratique commune de l'eau tout en légitimant l'action de l'administration dans l'ensemble des territoires concernés par la production et la mise en valeur de la ressource ${ }^{9}$. Au-delà, elle a implicitement pour vocation de reconstituer une unité territoriale que n’ont pas su créer les aménagements hydroagricoles.

Or l'activation de ce levier est entièrement déterminée par la perspective d'une pénurie, précisée dans son étendue et bornée dans le temps. C'est-à-dire que le changement au sein de l'hydrosystème est annoncé et agit, par anticipation, sur l'ensemble de la 
société. Dès lors, la solidarité n'est plus la traduction d'un sentiment partagé, mais apparaît comme une nécessité fondée sur un argumentaire scientifique et activée grâce à la menace que représente le futur. Elle est, en effet, d'autant plus légitime qu'elle s'appuie sur un fait présenté comme indépendant du fonctionnement social. Elle trouve d'abord sa traduction dans la responsabilisation de chaque individu vis-à-vis d'une société dont il n'est constitutif qu'à condition d'assumer sa dépendance à elle ; le contrat social est l'aboutissement de cette responsabilité partagée. L'État, investi du rôle de régulateur des liens sociaux au nom de l'équité générale, légitime ainsi un changement global des pratiques, une transformation de l'organisation institutionnelle de secteur de l'eau, l'instigation d'une tarification de l'eau et un aménagement grandissant de la ressource. La proposition faite pour résoudre les conflits régionaux entre territoires hydrauliques consiste donc en une surimposition d'un principe territorial supérieur (la solidarité) venant se substituer, d'une part, aux logiques propres à la petite et moyenne hydraulique et, d'autre part, au fonctionnement localisé des périmètres de grande hydraulique. D’une certaine manière, elle rend ainsi obsolète, car trop endogénéisée, la logique normative de l'hydraulique moderne. En d'autres termes, le nouveau succède à une nouveauté devenue héritage: c'est même là toute la légitimité du modèle appliqué.

Très concrètement, cette idéologie fortement empreinte d'une représentation caractéristique du devenir du territoire, transparaît à travers un certain nombre de marqueurs des concepts employés pour aménager le territoire (Di Méo, 1998). Ainsi, les zones de protection des bassins versants, les espaces concernés par la modernisation des réseaux de petite et moyenne hydraulique, sont motivés par la protection des grands équipements hydrauliques et de celle de la ressource. C'est aussi dans ces espaces que les premières tentatives de concertation et de participation des populations (grâce aux associations d'usagers) se mettent en place, nouvelle organisation censée offrir l'opportunité d'une adéquation entre les impératifs de l'action publique et de la réglementation en vigueur, avec les besoins locaux. L'ensemble de ces zones est marqué par l'évolution sémantique de la gestion de l'eau, chacune se voyant ainsi conférée la mission de mobiliser le collectif par l'emploi de techniques singulières : la réussite de cette mission réside dans la constitution d'un territoire défini sur un nouveau type de relation entre l'environnement, la population et l'administration.

En tentant d'effacer les spécificités territoriales, la mobilisation d'un objectif commun doit réduire l'ensemble des ordres de complexité (temporel, spatial, social, politique) au sein desquels s'est développée la crise. Ce mode d'action globalisant trouve son antonyme dans l'usage fait du temps pour la résolution du conflit autour du loup.

\section{Mobiliser du temps pour une gestion sociale du conflit}

Face à la divergence de représentations intervenant dans le cas du loup, l'action publique tente de mobiliser du temps pour faire converger les représentations du territoire dans le futur: il s'agit de compenser la perte de temps induite par l'adaptation à la présence du loup. Celle-ci nécessite en effet un surplus de gardiennage, la pose de filets pour permettre un regroupement quotidien du troupeau, mais aussi lors d'attaques, la recherche des brebis blessées ou mortes, pour percevoir une indemnisation. 
L'action publique nationale tente donc, dans le cadre limité imposé par la réglementation (protection de l'espèce), de compenser financièrement ou concrètement cette perte de temps, par ce qu'on peut appeler du temps fourni. L'État propose notamment le paiement d'heures supplémentaires de gardiennage ou l'emploi d'un aide berger. De même, au niveau départemental, les différents agents assermentés (de l'Office national de la chasse et de la faune sauvage - ONCFS -, des parcs nationaux, etc.) sont mobilisés pour effectuer les constats d'attaques nécessaires aux indemnisations. L'État mobilise ainsi du temps pour aider de manière financière et technique les éleveurs et bergers à s'adapter et à faire face à ces nouvelles contraintes. Enfin, du temps est consacré à la concertation au sein d'une instance nationale représentant les différentes parties concernées.

À la gestion sociale du conflit, s'ajoute également une dimension biologique. De nombreux agents de l'État assurent divers protocoles permettant de localiser et de dénombrer les populations de loups. Depuis 2004, une gestion directe du loup est menée à travers le tir mortel de quelques individus à l'origine de dégâts importants. Pour mener à bien ce protocole, des gardes ONCFS et des lieutenants de louveterie ont été mobilisés pendant plusieurs semaines, menant des affûts, généralement la nuit.

L’application locale de cette gestion montre néanmoins certaines disparités. C'est notamment le cas du Parc naturel régional du Vercors, gestionnaire de la Réserve naturelle des Hauts-Plateaux, située en son sein. La réserve, qui a connu le retour du loup en 1998, a en effet rapidement mis en place des systèmes simplifiant et accélérant les procédures nationales, par exemple celles de constat d'attaques. Le système départemental, déjà efficace, a été remplacé par un système spécifique aux Hauts-Plateaux: les gardes, présents en permanence sur le terrain, sont avertis d'une attaque très rapidement par les bergers, grâce aux radios que la réserve met à leur disposition. Ce dispositif permet par conséquent de raccourcir le délai d’intervention en matière de constat. Leur rôle ne s'arrête pas à la constatation de dégâts, car ils sont également présents auprès des bergers pour discuter, partager un repas, remplacer une radio défectueuse, etc.

De manière implicite, la réserve met à disposition du temps de gardes aux éleveurs et bergers, ce temps étant à la fois technique dans les procédures de constat et relationnel: lors du soutien psychologique faisant suite aux attaques, par exemple.

Enfin, les temps et les lieux officiellement consacrés au dialogue avec le monde agricole ont été non seulement pérennisés après l'arrivée du loup mais aussi complétés par une réunion annuelle de rencontre des partenaires du monde agricole (éleveurs, bergers) et du monde gestionnaire (parc naturel régional, réserve naturelle) sur les Hauts-Plateaux. C'est notamment la présence d'un territoire de projet qui facilite toute forme potentielle de dialogue (Mounet et Biron, 2003). Ce dialogue s'appuie sur un réseau de relations préexistant à l'arrivée du loup et structuré par la présence d'un tel territoire de projet. Cette possibilité de concertation prend toute son importance grâce à la présence des gardes qui, représentant des intermédiaires (Callon, 1992), facilitent la communication entre les gestionnaires et le monde agricole.

En parallèle à cette évolution de la gestion nationale et locale du conflit autour du loup, les positionnements des différentes parties prenantes se sont modifiés. Les éleveurs concernés par le loup ont peu à peu changé leurs pratiques, bien que cette évolution 
ne soit pas accompagnée d'un changement de discours. De même, les associations de protection de la nature ont évolué et certaines forment des bénévoles pour venir en aide aux éleveurs et bergers, notamment lors des estives.

Depuis une dizaine d'années, les camps qui s'affrontent au sujet du loup s’interpénètrent donc et on assiste à un mélange de savoirs exogènes et endogènes, spécifiques et génériques. Même si les désaccords persistent, les catégories définies en première partie pour lire le territoire montrent dans la réalité une hybridation de l'une par l'autre, au sens de Callon, Lascoumes et Barthe (2001). Celle-ci montre un glissement de la problématique du conflit. La complexité temporelle est résolue par un usage social et politique du temps: il permet d'atténuer le caractère prescriptif du territoire des lycophiles, par la construction d'une action individualisée auprès de l'éleveur.

\section{Conclusion}

La grille de lecture adoptée initialement permet de distinguer non seulement des types de temps différents intervenant dans la crise, mais aussi des temps mobilisés par ou pour des échelles et des groupes distincts. Dans les deux cas étudiés, le conflit voit la confrontation des temps référents élaborés par les différents groupes sociaux concernés, et relevant de leurs représentations distinctes de l'histoire collective. Le temps qui fait conflit est donc dans les deux cas de l'ordre de la temporalité des acteurs. L'échelle sociale concernée par ce temps, vecteur de conflit, se situe au niveau du groupe. Face à cela, les exemples abordés montrent deux types de réponse, portant sur une échelle différente (figure 1). Dans le cas du loup, la mobilisation, par l'action publique, d'un temps utilisable par l'éleveur et le berger correspond à une formulation de la réponse à l'échelle de l'individu ; mais surtout le temps convoqué est d'une toute autre nature que celui qui fait conflit: il s'agit bien d'un temps concret et utilisable et non d'une temporalité. Dans le cas de la gestion de l'eau, l'argument d'un principe supérieur commun, chevillé à la perspective d'une échéance commune, celle de la pénurie, renvoie à un temps partagé non plus par le groupe, la communauté, mais par la société.

\section{Figure 1 Nature et échelle du temps mobilisé dans la résolution du conflit}

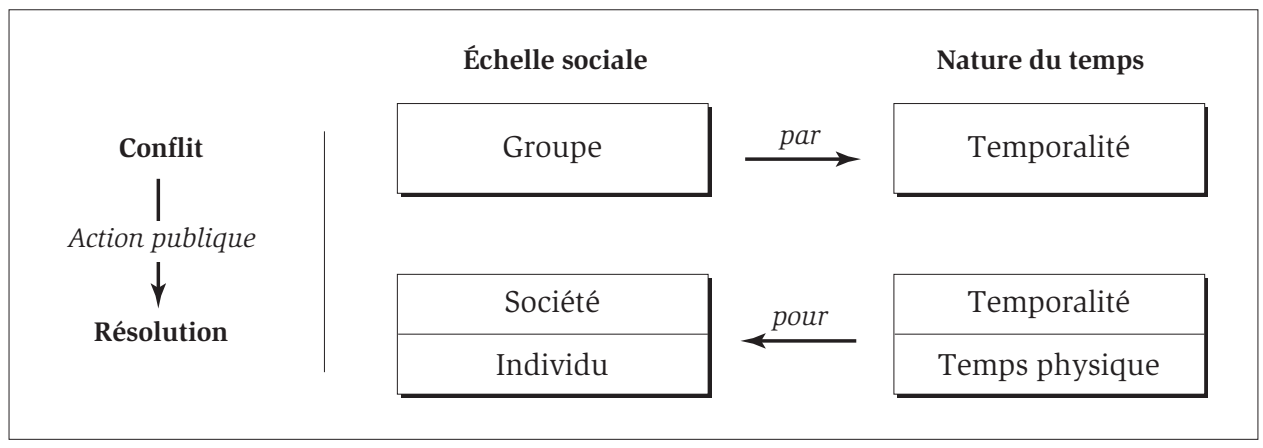

Si, dans les deux cas, l'action publique est le moteur du changement des pratiques, elle n'emploie pas les mêmes vecteurs. Or c'est en déterminant leur nature qu'il va être possible de dire s'ils participent ou non à un processus de territorialisation: permettent-ils 
une nouvelle appropriation du territoire? Des disparités apparaissent entre les deux situations étudiées. Si l'on considère qu'un processus de territorialisation doit articuler une dimension organisationnelle, une dimension spatiale et une dimension sociale, les vecteurs employés pour la gestion du loup et celle de l'eau ne mobilisent pas de manière égale ces trois dimensions. Dans le cas du loup, la gestion du conflit revêt des aspects organisationnels et sociaux d'ordre relationnel. Les moyens temporels mobilisés et fournis au monde agricole permettent le fonctionnement des nouvelles pratiques. L'aspect relationnel, quant à lui, recouvre les temps formels et informels consacrés au dialogue. Ce dernier aspect prend une place plus importante dans le cas de la gestion menée par la Réserve naturelle des Hauts-Plateaux et le Parc national régional du Vercors. Ceux-ci permettent en effet d'accompagner et d'aménager localement le changement de pratiques organisé nationalement. La mobilisation de l'aspect relationnel facilite donc l'appropriation d'une nouvelle norme sociale intégrant, dans les pratiques et non dans les représentations, la présence du loup. Mais il est nécessaire de laisser faire le temps pour que les représentations s'accordent aux pratiques.

Dans le cadre de la gestion de l'eau, le lien que constitue le réseau hydraulique permet en revanche une structuration forte de l'espace en fonction des enjeux propres au territoire: il incarne le système d'idées déployé par le gestionnaire. S'il n'y a pas nécessairement appropriation du territoire, il y a en revanche diffusion d'une idéologie territoriale qui permet d'orienter l'ensemble des actions des groupes sociaux.

On retrouve dans les deux cas une transformation organisationnelle, induisant ellemême une réorganisation spatiale; mais ces deux aspects ne revêtent pas les mêmes réalités. Dans le cas de l'eau, l'aspect organisationnel renvoie à la modification du système de fonctionnement alors que dans celui du loup, il renvoie à l'invention d'une nouvelle organisation. La transformation spatiale se traduit dans un cas, par un aménagement physique et visible alors que dans l'autre, bien qu'elle soit évidente (liée aux zones occupées par le loup), elle reste ténue et correspond à l'adoption de nouvelles pratiques.

Si ces deux aspects représentent des vecteurs structurants du territoire pour le loup et l'eau, il reste néanmoins difficile de parler à ce sujet de territorialités propres. L’appropriation idéelle par les sociétés locales de ces nouvelles normes demande en effet un certain recul. Cette situation soulève une nouvelle hypothèse: en se faisant sur le temps, l'action reste abstraite et moins contraignante que peut l'être une action sur l'espace. Dans ce sens, en s'appliquant à l'épiphénomène sans réellement traiter le problème des divergences de représentation, l'action contourne la complexité plus qu’elle ne la réduit.

\section{Notes}

1 Cet article présente un des résultats du séminaire «Formes et enjeux des temporalités dans la construction collective des territoires» (2004/2006) de l'équipe Territoires, au sein de l'Unité Mixte de Recherche «Pacte» (Université Joseph Fourier/Université Pierre Mendès, Grenoble, le Centre National de la Recherche Scientifique (CNRS) et l'Institut d'Études Politiques (IEP)). Ce séminaire exploratoire s'est intéressé aux implications du temps dans la construction des territoires, particulièrement dans les champs laissés libres par 
les approches de la time geography et de l'étude de la multiplication des rythmes sociaux, individuels et collectifs. La bibliographie présentée en fin de texte ne fait donc mention que d'ouvrages généraux à partir desquels s'est construite la démarche intellectuelle de ce travail collectif.

2 On définit la crise comme un état de rupture, de déséquilibre entre un état ancien qui n'est plus adapté à la nouvelle situation et un état nouveau à définir. La crise, provoquée par une pénurie de sens, de revenus, de moyens intellectuels et matériels, débouche sur une régression, sur une évolution ou sur un conflit (Bréard et Pastor, 2000). Par système, on entend un ensemble d'éléments en interactions fortes, structurées, organisées et relativement stables (Gumuchian et al., 2003).

3 On définit le conflit comme la rencontre d'intérêts contraires qui se traduit, dans les exemples traités, par une compétition pour des ressources rares (l'eau, l'espace), par des tendances à l'autonomie et la divergence entre les objectifs des différents acteurs (Pondy, 1967).

4 La notion de temporalité utilisée ici renvoie à un usage phénoménologique du temps, tel que les groupes sociaux et les individus se le représentent, et non pas à une période ou à un partage du temps.

5 Pour plus de détails sur cette question, voir Alexandre, 2004.

6 Pour plus de détails sur cette question, voir Mounet, 2007.

7 Leur analyse se fonde sur deux types de corpus: la littérature grise (rapports d'études, expertises, documents programmatifs et évaluations émis par les acteurs et les opérateurs) et les politiques publiques établies dans les domaines concernés; des entretiens semidirectifs auprès des acteurs publics de l'eau au Maroc et auprès des différents acteurs pris dans le conflit du loup.

8 Par apodictique, on entend l'émergence d'une entité territoriale fondée sur une évidence de droit et non pas seulement de faits, comme un résultat donné a priori par la démonstration et non par l'expérience. Inversement, l'entité assertorique se fonde sur une évidence de faits, d'expérience.

9 Pour plus de précisions, voir Alexandre, 2005. 


\section{Bibliographie}

ALEXANDRE, Olivier (2004) Le territoire dans les stratégies de gestion de l'eau. De l'intégration sectorielle à l'intégration des territoires au Maroc. Géocarrefour, vol. 78, $\mathrm{n}^{\circ} 2$, pp. 99-107.

ALEXANDRE, Olivier (2005) Lorsque la pénurie fait ressource. Étude géographique de la notion de ressource. L'exemple de la politique de l'eau au Maroc. Géocarrefour, vol. $80, n^{\circ} 4$, pp. $263-271$.

ASCHER, François et GODARD, Francis (dir.) (2003) Modernité: la nouvelle carte du temps. La Tour d'Aigues, Éditions de l'Aube.

BERTRAND, Claude et BERTRAND, Georges (2000) Le géosystème; espace-temps anthropisé. Esquisse d'une temporalité environnementale. Dans Monique BarruePastor et Georges Bertrand (dir.) Les temps de l'environnement, Toulouse, Presses universitaires du Mirail, pp. 65-76.

BOULIN, Jean-Yves (dir.) (2002) La nouvelle aire du temps. La Tour d'Aigues, Éditions de l'Aube.

BRÉARD, Richard et PASTOR, Pierre (2000) Gestion des conflits: la communication à l'épreuve. Rueil-Malmaison, Éditions Liaisons.

CALLON, Michel (1992) Sociologie des sciences et économie du changement technique: l'irrésistible montée des réseaux technico-économiques. Dans Bruno Latour (dir.) Ces réseaux que la raison ignore, Paris, L'Harmattan.

CALLON, Michel, LASCOUMES, Pierre et BARTHE, Yannick (2001) Agir dans un monde incertain. Essai sur la démocratie technique. Paris, Seuil.

CHESNEAU, Jean (2000) Temps de l'histoire naturelle et temps de l'histoire humaine. Dans Monique Barrue-Pastor et Georges Bertrand (dir.) Les temps de l'environnement, Toulouse, Presses universitaires du Mirail, pp. 33-39.

COLLECTIF (1983) L'espace et le temps aujourd'hui. Paris, Seuil.
DA COSTA GOMÈS, Paolo César (1997) Les deux pôles épistémologiques de la modernité. Une lecture des fondements de la géographie chez Kant et Herder. Dans Jean-François Staszak (dir.) Les discours du géographe, Paris, L'Harmattan.

DI MÉO, Guy (1998) Géographie sociale et territoires. Paris, Nathan.

DOLLFUS, Olivier, GRATALOUP, Christian et LÉVY, Jacques (1999) Le Monde: pluriel et singulier. Dans Michel Beaud, Olivier Dollfus, Christian Grataloup, Philippe Hugon, Gérard Kébabdjian et Jacques Lévy (dir.) Mondialisation: les mots et les choses, Paris, Karthala.

GUMUCHIAN, Hervé, GRASSET, Éric, LAJARGE, Romain et ROUX, Emmanuel (2003) Les acteurs, ces oubliés du territoire. Paris, Anthropos.

LARRÈRE, Catherine (1997) Les philosophies de l'environnement. Paris, Presses universitaires de France.

LASCOUMES, Pierre (1994) L'éco-pouvoir, environnements et politiques. Paris, La Découverte.

MARIÉ, Michel (1982) Un territoire sans nom. Pour une approche des sociétés locales. Paris, Librairie des Méridiens.

MAUZ, Isabelle (2002) Gens, cornes et crocs. Relations hommes-animaux et conceptions du monde, en Vanoise, au moment de l'arrivée des loups. Thèse de doctorat, Sciences de l'environnement, ENGREF, Paris.

MICOUD, André (1993) Vers un nouvel animal sauvage, le sauvage "naturalisé vivant»? Natures, Sciences et Sociétés, vol. 3, n 1 , pp. 202-210.

MOUNET, Coralie (2007) Les territoires de l'imprévisible. Conflits, controverses et "vivre ensemble» autour de la gestion de la faune sauvage. Le cas du loup et du sanglier dans les Alpes françaises. Thèse de doctorat de géographie, Université Grenoble I. 
MOUNET, Coralie et BIRON, Pierre-Eymard (2003) Le loup dans le Vercors: gestion concertée ou "négociée»? Montagnes Méditerranéennes, vol. 18, pp. 39-45.

PONDY, Louis R. (1967) Organizational Conflict: Concepts and Models. Administrative Science Quarterly, vol. 12, pp. 296-320. 